\title{
$\rightarrow$ \\ Fecal incontinence, an ignored and concealed problem
}

Fecal incontinence is a symptom on which gastroenterologists are poorly informed, whereas an understanding of this complaint may allow clinicians to provide help for most patients $(1,2)$. It is an involuntary, recurrent loss of feces through the anus (3). It is called anal incontinence when there is an unwanted release of gas, whether or not in association with fecal loss. Individuals suffering from this condition experience a deeply negative impact on their quality of life both in the physical and psychological spheres, which accounts for the increasingly greater attention they receive. The true prevalence of fecal incontinence is unknown, since those who experience it will not usually disclose this symptom. It is believed to involve approximately $5 \%$ of the general population $(4,5)$, and almost $50 \%$ of elderly individuals in geriatric nursing homes $(6,7)$. Its frequency is supposed to become higher in the next few years, given the population's longer life expectancy. In this issue of Revista Española de Enfermedades Digestivas, Ballester et al. (8) review the presence of anal and urinary (double) incontinence in women attending a primary care clinic for other reasons, and discuss factors related thereto. To this end they evaluate 103 women between 20 and 64 years of age with no known risk factors. They found that the prevelence of double incontinence was $9.7 \%$, similar to that found in other studies (9) and in a Spanish study on urinary incontinence (10). Although including a moderate number of patients, this is the only study of this type ever performed in Spain, and as we just mentioned reveals that the prevalence of incontinence has been underestimated.

Fecal incontinence develops when structures or functions in the anal-rectal region become altered (fecal consistency, anatomic and neurologic integrity of anal sphincters, rectal compliance and sensitivity) (11-13). The most common causes that may impair these mechanisms include diarrhea, inapparent injury during birth, and neurologic conditions. It is a common occurrence that following years of normal continence women develop incontinence during the sixth or seventh decade of life when aging and hence pudendal nerve denervation and sphincter muscle weakness act on previous obstetric lesion (14-16). Diarrhea usually unveils, favors or aggravates some other disordered mechanism of fecal retention (2). Regarding therapeutic decision making, it is important that true diarrhea be told apart from false, overflow diarrhea. The latter is common in the elderly as a result of fecal impactation from limited physical status, which induces relaxation of the internal anal sphincter and hence potential soiling. Chronic constipation may in addition result in external anal sphincter hipotonia through pudendal nerve injury during defecation stress (17).

Elderly patients with fecal incontinence are known to also experience urinary incontinence; hence it is considered that having one type of incontinente (either 
fecal or urinary) is a risk factor for having the other type (18). A close association has been reported between double incontinence and vaginal delivery or chronic regional stress, but few studies have investigated factors predisposing to double incontinence in the female general population $(10,19)$. As Ballester et al. conclude in this issue of Revista Española de Enfermedades Digestivas (8), age, number of vaginal deliveries, and overweight are associated with the presence of anal and/or urinary dysfunction. Although age and the number of vaginal deliveries have been consistently reported in the literature as risk factors for incontinence, such is not the case for overweight (20). Results obtained by these indicate suggest that overweight is an independent risk factor for both anal and urinary incontinence in women, and they suggest that greater weight may result in extremely high intraabdominal pressures, which would in turn impair the pelvic floor (21).

Symptoms and a physical examination help intuit some causal mechanisms on occasion (22); however, having recourse to other, more complex diagnostic techniques is often needed (23). Although to date no protocol has been firmly established for the assessment of anorectal conditions, techniques currently recommended for the exploration of fecal incontinence include anorectal manometry, rectal sensitivity testing, surface electromyography, and ultrasonography $(4,24,25)$. These tests complement each other, as the etiology of fecal incontinence is usually multiple. With the information provided by these tests the mechanisms involved in continence and defecation may be better understood, and more rational therapies may be initiated (26-28). However, the fact that they are performed under non-physiologic conditions is somehow a limitation.

The information provided by these studies is essential for treatment selection; indeed, if a structural sphincteric disorder is revealed, this may be amenable to surgical treatment, whereas other etiologies may benefit from conservative therapy. Regarding the latter, any causes of diarrhea should be controlled using specific measures. Otherwise, hygienic-dietary recommendations, bulking agents, and or antidiarrheic agents such as loperamide may be indicated. The mechanism through which antidiarrheic agents act is thought to be by slowing down bowel motility and by decreasing stooling frequency, which would result in better formed feces. Aminotryptiline is a tricyclic antidepressant that has been empirically used to improve symptoms in patients with idiopathic fecal incontinence or diarrhea-predominant irritable bowel syndrome. In low doses it reduces rectal motility, most probably through an anticholinergic action (29). Besides being useful against diarrhea, loperamide increases the tonus of the internal anal sphincter (30). Phenylephrine gel is undergoing clinical trials, as it plays a role in smooth muscle vascularization and hence modulates internal anal sphincter innervation, increases sphincteric tonus, and may be useful for patients with passive fecal incontinence, who have an intact though hypotonic internal anal sphincter. Side effects are minimal, and its usefulness for structural disorders in this sphincter is being assessed (31). In case of overflow diarrhea the rectum should be lavaged using suppositories or enemas, and laxatives should be prescribed. In the elderly hygienic-dietary measures and drug therapies may help improve their status during this time of life. Biofeedback techniques attempting to strengthen the external sphincter or to improve rectal sensitivity may be associated with these measures (32). This may be result in improvement in around $75 \%$ of patients, and in healing in around $50 \%$ of cases $(2,33)$. However, the real usefulness of this technique has not been established yet, since reported studies about it are non-controlled, and 
patient selection, biofeedback type and duration, and response assessment lacked homogeneous criteria. On the other hand the role played by other concurrently prescribed therapies awaits definition (33). In this sense the patient-physician relationship regarding education on useful strategies has been seen to be potentially more relevant for the improvement of fecal incontinence than exercise or biofeedback on sphincteric function (32). The fact that the etiology of fecal incontinence is often multiple makes it possible that continence be achieved by merely correcting some the involved causes. Finally, sacral nerve stimulation has shown promising preliminary results with minimal morbidity; however, few studies are available thus far to define its role in the management of fecal incontinence (34).

In view of the common occurrence of both fecal and urinary incontinence in one patient, studies on both disorders should be carried out by interdisciplinary teams (gastroenterologists, surgeons, urologists, gynecologists, physical therapists, psychologists). The therapies for fecal incontinence (biofeedback, sphincteroplasty, antidiarrheic agents, laxatives, sacral nerve stimulation) require validation from controlled randomized studies. Regarding urinary incontinence, a comparison of drug, behavioral, and surgical therapies is still due. Similarly, studies comparing combination therapies using biofeedback and surgery versus surgery alone or biofeedback alone are also needed. New drugs acting on baseline anal pressure are needed for fecal incontinence, and other drugs acting on distension hypersensitivity would be most welcome for urinary urge incontinence (35).

The incidence of incontinence may probably be significantly decreased if risk factors are identified and excluded. For instance, a number of standard obstetric practices such as unnecessary episiotomy or cesarean delivery in high-risk patients should be avoided, and women should be instructed to work up their pelvic floor before and after delivery, and educated to avoid stress during defecation. Similarly, since a number of authors - including Ballester et al. in their paper in this issue (8) - conclude that overweight is a risk factor for incontinence, it seems reasonable that preventive measures are taken against it, particularly given the fact that obesity is becoming increasingly common in the general population.

In addition, understanding and informing these patients, who may sometimes need psychological counseling, may help them get over the social impact of this improvable disabling condition, which is challenging for physicians and leaves a wide margin for further investigation.

M. T. Muñoz Yagüe

Service of Digestive Diseases. Hospital Universitario 12 de Octubre. Madrid, Spain

\section{REFERENCES}

1. Whitehead WE. Fecal incontinence: a neglected area of Gastroenterology. Comment from the editors. Gastroenterology 2002; 122: 5 .

2. Whitehead WE, Wald A, Norton NJ. Treatment options for fecal incontinence. Dis Colon Rectum 2001; 44: $131-44$.

3. Whitehead WE, Wald A, Diamand NE, Enk P, Pemberton J, Rao SS. Functional disorders of the anus and rectum. Gut 1999; 45: 1155-9. 
4. Diamand NE, Kamm MA, Wald A, Whitehead WE. AGA technical review on anorectal testing techniques. Gastroenterology 1999; 116: 732-60.

5. Drossman DA, Li Z, Andruzzi E, Temple RD, Talley NJM, Thompson WG, et al. US householder survey of functional gastrointestinal disorders. Prevalence, sociodemography, and health impact. Dig Dis Sci 1993; 38: 1569-80.

6. Nelson RL, Furner S, Jesudason V. Fecal incontinence in Wisconsin nursing homes. Prevalence and associations. Dis Colon Rectum 1998; 41: 1226-9.

7. Nelson RL. Epidemiology of fecal incontinence: Dimensions of the problem: prevalence and impact. Gastroenterology 2004; 126: S3-S7.

8. Ballester A, Mínguez M, Herreros B, Hernández V, Sanchiz V, Benages A. Prevalencia de la incontinencia anal y urinaria silentes en mujeres de la ciudad de Teruel. Rev Esp Enf Dig 2005; 97: 78-86.

9. Siracusano S, Pregazzi R, d'Aloia G, Sartore A, Di Benedetto P, Pecorari V, et al. Prevalence of urinary incontinente in young and middle-aged women in an Italian urban area. Eur J Obstet Gynecol Reprod Biol 2003; 107: 201-4.

10. Lacima G, Espuña M, Pera M, Puig-Clota M, Quinto L, García-Valdecasas JC. Clinical, urodynamic, and manometric findings in women with combined fecal and Urinary incontinente. Neurourol Urodyn 2002; $21: 464$ 9.

11. Cooper ZR, Rose S. Fecal incontinence: a clinical approach. Mt Sinai J Med 2000; 67: 96-105.

12. Smouth AJP, Akkermans LMA. Rectum, anus and pelvic floor. En: Normal and disturbed motility of the gastrointestinal tract. U.K. Petersfield Hampshire: Wrightson Biomedical Publishing Ltd (Ed.) 1992. p.169-210.

13. Sagar PM, Pemberton JH. Anorrectal and pelvic floor function. Relevance to continence, incontinence and constipation. Gastroenterol Clin North Am 1996; 25: 163-82.

14. Laurberg S, Swash H. Effects of aging on the anorectal sphincters and their innervation. Dis Colon Rectum 1989; 32: 737-42.

15. Kamm MA. Obstetric damage and fecal incontinence. Lancet 1994; 344: 730-3.

16. Rasmussen O, Christiansen T, Tetzschener T, Sorensen M. Pudendal nerve function in idiopathic fecal incontinence. Dis Colon Rectum 2000; 43: 633-7.

17. Parks AG, Swash M, Urich H. Sphincter denervation in anorectal incontinence and rectal prolapse. Gut 1977; 18: 656-65.

18. Roberts RO, Jacobsen SJ, Reilly WT, Pemberton JH, Lieber MM, Talley NJ. Prevalence of combined fecal and urinary incontinence: a community-based study. J Am Geriatr Soc 1999; 47: 837-41.

19. Lacima G, Pera M. Combined fecal and urinary incontinence an update. Current Opinion in Obstetric Gynecology 2003; 15: 405-10

20. Fornell ES, Wingren G, Kjolhede P. Factors associated with pelvic floor dysfunction with emphasis on urinary and fecal incontinence and genital prolapse: an epidemiological study. Acta Obstet Gynecol Scand 2004; 83: 383-9.

21. Noblett KL, Ostergard DR. The relationship of body mass index to intra-abdominal pressure as measured by multichannel cystometry. Int Urogynecol J Pelvic Floor Dysfunct 1997; 8: 323-6.

22. Hill J, Corson RJ, Brandon H, Redford J, Farager EB, Kiff ES. History and examination in the assessment of patients with idiopatic fecal incontinence. Dis Colon Rectum 1994; 37: 473-7.

23. Azpiroz Vidaur F. Guía práctica sobre incontinencia anal. Rev Esp Enferm Dig 2003; 95: 717-21.

24. Azpiroz F, Enk P, Whitehead WE. Anorectal function testing. Review of collective experience. Am J Gastroenterol 2002; 97: 232-40.

25. Muñoz Yagüe T, Álvarez Sánchez V, Ibáñez Pinto A, Solís-Herruzo JA. Clínica, manometría anorrectal y electromiografía de superficie en el estudio de los pacientes con incontinencia fecal. Rev Esp Enferm Dig 2003; 95: 629-34.

26. Smith Lee E. Practical guide to anorectal testing. $2^{\text {nd }}$ ed. New York-Tokyo: Igaku-Shoiw, 1995.

27. Mínguez M, Benages A. Alteraciones motoras anorrectales. En: Ponce J, ed. Motilidad Digestiva II. Función Normal. Métodos de Estudio. Barcelona-Philadelphia: Prous Science, 1996. p. 323-43.

28. Azpiroz Vidaur F. Progresos en Gastroenterología. Utilidad clínica de la evaluación de la función anorrectal. Gastroenterol Hepatol 1998; 21: 294-301.

29. Scarlett Y. Medical management of fecal incontinence. Gastroenterology 2004; 126: S55-S63.

30. Read M, Read NW, Barber DC, Duthie HL. Effects of loperamide on anal sphincter function in patients with fecal incontinence and urgency. Dig Dis Sci 1982; 27: 807-14.

31. Cheetham MJ, Kamm MA, Phillips RKS. Topical phenilephrine increases anal canal resting pressure in patients with faecal incontinence. Gut 2001; 48: 356-9.

32. Norton C. Behavioral management of fecal incontinence in adults. Gastroenterology 2004; 126: S64-70.

33. Heimen S, Jones KR, Ringel Y, Scarlett Y, Whitehead WE. Biofeedback treatment of fecal incontinence. Dis Colon Rectum 2001; 44: 728-36.

34. Madoff RD. Surgical treatment options for fecal incontinence. Gastroenterology 2004; 126: S48-S54.

35. Whitehead WE, Wald A, Norton NJ. Priorities for treatment research from different professional perspectives. Gastroenterology 2004; 126: S180-85. 


\section{Incontinencia fecal, un problema ignorado y encubierto}

La incontinencia fecal constituye una manifestación sobre la que los gastroenterólogos están poco informados y en la que la mayoría de los pacientes pueden ser ayudados si se tienen ciertos conocimientos sobre ella $(1,2)$. Consiste en la pérdida involuntaria y recidivante de heces por el ano (3). Cuando existe escape de gas, asociado o no al de heces, hablamos de incontinencia anal. Las personas que la padecen ven afectada muy negativamente su calidad de vida tanto en el aspecto físico como en el psicológico, lo que justifica que se les preste una atención cada vez mayor. Su prevalencia real es desconocida ya que, quienes la sufren, con frecuencia ocultan su existencia. Se cree que afecta aproximadamente al $5 \%$ de la población general $(4,5)$ y hasta a casi el 50\% de los ancianos ingresados en instituciones geriátricas (6,7). Se supone que su frecuencia aumentará en los próximos años debido a la mayor supervivencia de la población. En este número de la Revista Española de Enfermedades Digestivas, Ballester y cols. (8) investigan la presencia de incontinencia anal y urinaria (doble incontinencia) en mujeres que acuden a una consulta de Atención Primaria por motivos distintos a ello y analizan los factores relacionados con ella. Con este fin, evalúan 103 mujeres con edades comprendidas entre los 20 y los 64 años sin factores de riesgo conocidos. Encuentran que la prevalencia de la doble incontinencia es del 9,7\%, similar a la hallada en otros estudios (9) y al realizado en España en pacientes con incontinencia urinaria (10). Si bien se trata de un estudio que incluye un número de pacientes no grande, se trata del único de estas características realizado en España y que pone de manifiesto, como hemos referido, que la prevalencia de la incontinencia está subestimada.

La incontinencia fecal se origina cuando las estructuras o las funciones de la región ano-rectal que intervienen en la continencia se alteran (consistencia de las heces, integridad anatómica y neurológica de los esfínteres anales, "complianza" y sensibilidad rectales) (11-13). Entre las causas más frecuentes que pueden alterar los mecanismos anteriores se encuentran la diarrea, las lesiones inaparentes producidas durante el parto y las de origen neurológico. En las mujeres, es frecuente que, tras años de continencia normal, la incontinencia se manifieste al llegar a la $6^{\mathrm{a}} \mathrm{o} 7^{\mathrm{a}}$ décadas de la vida, cuando sobre una lesión obstétrica antigua se superpone el envejecimiento general con la consiguiente denervación del nervio pudendo y la debilidad de los músculos esfinterianos (14-16). La diarrea suele poner de manifiesto, favorecer o agravar algún otro trastorno del mecanismo de retención de las heces (2). Con vistas a la actitud terapéutica a tomar, es importante diferenciar una diarrea verdadera de una falsa diarrea o "por rebosamiento". Esta última es frecuente en los ancianos, en quienes, debido a sus limitadas condiciones físicas, se produce una impactación fecal, la cual origina la relajación del esfínter anal interno y, por lo tanto, la posibilidad del escape. El estreñimiento crónico puede, además, provocar la hipotonía del esfínter anal externo por lesión del nervio pudendo durante los esfuerzos defecatorios (17).

Se ha comprobado que los ancianos con incontinencia fecal con frecuencia padecen también incontinencia urinaria, de forma que, se considera que padecer uno de los tipos de incontinencia (fecal o urinaria) constituye un factor de riesgo para sufrir el otro (18). Se ha referido que existe una estrecha asociación entre la doble incontinencia con la historia de partos vaginales y con los esfuerzos crónicos, pero existen 
pocos estudios que hayan analizado los factores predisponentes de doble incontinencia en la población general femenina $(10,19)$. Tal como concluyen Ballester y cols. en este número de la Revista Española de Enfermedades Digestivas (8), la edad, el mayor número de partos vaginales y el sobrepeso se asocian con la presencia de disfunción anal y/o urinaria. Si bien la edad y el número de partos vaginales son factores de riesgo para la incontinencia referidos de forma reiterada en la literatura, no lo es tanto el sobrepeso (20). Los resultados obtenidos por estos autores indican que el sobrepeso en las mujeres constituye un factor de riesgo independiente tanto para la incontinencia anal como para la urinaria y sugieren que el mayor peso condicionaría la existencia de presiones intraabdominales muy elevadas que provocarían la lesión del suelo pélvico (21).

En ocasiones, la sintomatología y la exploración física ayudan a intuir algunos de los mecanismos causales (22) pero, en otros casos, es necesario recurrir a técnicas diagnósticas más complejas (23). Aunque en la actualidad no existe un protocolo establecido para la valoración de la fisiopatología ano-rectal, las técnicas exploratorias actualmente recomendadas para la incontinencia fecal son la manometría ano-rectal, las pruebas de sensibilidad rectal, la electromiografía de superficie y la ultrasonografía $(4,24,25)$. Se trata de pruebas complementarias entre sí, ya que con frecuencia la etiología de la incontinencia fecal no es única. Con la información que proporcionan se obtiene un mejor conocimiento de los mecanismos que intervienen en la continencia y en la defecación y se pueden indicar tratamientos más racionales (26-28). Sin embargo, el hecho de que se practiquen en condiciones no fisiológicas constituye una cierta limitación.

La información que aportan estos estudios es fundamental para indicar el tipo de tratamiento, ya que si se concluye que existe una alteración estructural esfinteriana, esta puede ser susceptible de tratamiento quirúrgico, mientras que otras causas pueden beneficiarse de un tratamiento conservador. En relación con este último, si existe una causa de la diarrea, esta debe ser controlada con el tratamiento específico. Si no la hay, se pueden indicar medidas higiénico-dietéticas, agentes "formadores de masa" y/o antidiarreicos como la loperamida. Se cree que el mecanismo por el que actúan los antidiarreicos es enlenteciendo la motilidad intestinal y disminuyendo la frecuencia de las deposiciones con lo que resultarían unas heces más formadas. La aminotriptilina es un antidepresivo tricíclico que ha sido utilizado empíricamente para mejorar los síntomas de pacientes con incontinencia fecal idiopática o con síndrome de intestino irritable con predominio de la diarrea. En dosis bajas, disminuye la motilidad rectal, probablemente a través de un mecanismo anticolinérgico (29). La loperamida, además de tratar la diarrea, aumenta el tono del esfínter anal interno (30). El gel de fenilefrina está siendo probado en estudios clínicos ya que interviene en la vascularización del músculo liso y, por ello, modula la inervación del esfínter anal interno, aumenta su tono y puede ser útil en pacientes con incontinencia fecal pasiva que tienen un esfínter anal interno intacto pero hipotónico. Los efectos secundarios son mínimos y se está empezando a valorar su utilidad en los defectos estructurales de este esfínter (31). Si la diarrea es "por rebosamiento", se debe hacer limpieza del recto mediante supositorios o enemas e indicar laxantes. En los ancianos, las medidas higiénico-dietéticas y los tratamientos farmacológicos pueden ayudar a mejorar la situación de esta etapa de la vida. A todas estas medidas se puede asociar la práctica de "biofeedback" con el que se intenta fortalecer el esfínter anal externo o bien mejorar la sensibilidad rectal (32). Con ello se puede lograr la mejoría en aproximadamente el $75 \%$ de los casos y su curación en torno al 50\% $(2,33)$. Sin embargo, la utilidad real de esta técnica está pendiente de ser determinada ya que los estudios de que se dispone no son controlados y la selección de los pacientes, la duración y el tipo de "biofeedback" así como la valoración de la respuesta no se han realizado con criterios homogéneos. Por otro lado, queda por definir el papel que juegan en la respuesta que se obtiene las restantes medidas terapéuticas que se 
indican simultáneamente (33). En este sentido, se ha comprobado que la relación entre el paciente y el médico para el aprendizaje de estrategias puede ser más importante para el logro de la mejoría de la incontinencia fecal que la realización de ejercicios o el recibir "biofeedback" de la función esfinteriana (32). El hecho de que con frecuencia la etiología de la incontinencia fecal sea múltiple hace que pueda lograrse la continencia fecal corrigiendo sólo alguna de sus causas. Finalmente, la estimulación del nervio sacro ha mostrado resultados provisionales prometedores y origina una mínima morbilidad, sin embargo, hasta el momento disponemos de pocos estudios para que nos definan su papel en el tratamiento de la incontinencia fecal (34).

Debido a la frecuencia con que la incontinencia fecal y la urinaria coexisten, los estudios de ambos desórdenes deben ser realizados por grupos multidisciplinares (gastroenterólogos, cirujanos, urólogos, ginecólogos, fisioterapeutas, ginecólogos, psicólogos). Los tratamientos para la incontinencia fecal ("biofeedback", esfinteroplastia, antidiarreicos y laxantes y estimulación del nervio sacro) precisan ser validados por estudios controlados y randomizados. En relación con la incontinencia urinaria, quedan pendientes de comparar los tratamientos farmacológicos, conductual y quirúrgicos. Son asimismo necesarios estudios que valoren los tratamientos combinados con "biofeedback" y cirugía, cirugía únicamente o "biofeedback" aislado. Se precisan nuevos fármacos que actúen a nivel de la presión basal del ano en la incontinencia fecal y sobre la hipersensibilidad a la distensión en la incontinencia urinaria de urgencia (35).

Es probable que pueda reducirse de forma significativa la incidencia de la incontinencia identificando y evitando factores de riesgo. Por ejemplo, se deben modificar determinadas prácticas obstétricas habituales como es evitar las episiotomías innecesarias o bien practicar cesáreas a las pacientes de alto riesgo, además de indicar ejercicios del suelo pélvico antes y después del parto y educar a los pacientes para eludir los esfuerzos durante la defecación. Asimismo, dado que algunos autores, entre los que se encuentra Ballester y cols. en el artículo comentado en este número de la revista (8), concluyen que el sobrepeso constituye un factor de riesgo para la incontinencia, parece razonable tomar medidas preventivas para evitarlo, más aún teniendo en cuenta el aumento progresivo de la frecuencia de la obesidad en la población general.

Además, la comprensión e información de estos pacientes, que en ocasiones precisan ayuda psicológica, puede ayudarles a superar la repercusión social de esta situación invalidante que puede ser mejorada, constituye un reto para el médico que se enfrenta a ella y en la que queda un amplio campo por investigar.

M. T. Muñoz Yagüe

Servicio de Medicina de Aparato Digestivo. Hospital Universitario 12 de Octubre. 